\title{
Heavy Meson-Antimeson Molecules in Effective Field Theory: the Role of One Pion Exchange and Heavy Quark Spin Symmetry
}

\author{
M. Pavón Valderrama* \\ Instituto de Física Corpuscular (IFIC), Centro Mixto CSIC-Universidad de Valencia, \\ Institutos de Investigación de Paterna, Apartado 22085, E-46071 Valencia, Spain \\ E-mail: m.pavon.valderrama@ific.uv.es
}

\section{J. Nieves}

Instituto de Física Corpuscular (IFIC), Centro Mixto CSIC-Universidad de Valencia, Institutos de Investigación de Paterna, Apartado 22085, E-46071 Valencia, Spain

\begin{abstract}
In this contribution we study heavy meson-antimeson molecules from the effective field theory perspective. In particular, we are concerned with the role of the one pion exchange interaction and heavy quark spin symmetry in the description of molecular states. As we will show, one pion exchange is in general a perturbative effect. This means that at lowest order we can describe the molecular states in terms of contact interactions only, a remarkable simplification. The application of heavy quark spin symmetry will be very interesting, as we will be able to predict new heavy meson-antimeson bound states from known ones. For instance, if we assume that the $X(3872)$ is in fact a $J^{P C}=1^{++} \mathrm{D}^{*} / \mathrm{D}^{*} \overline{\mathrm{D}}$ molecule, we can predict the existence of a $J^{P C}=2^{++} \mathrm{D}^{*} \overline{\mathrm{D}}^{*}$ molecular partner with a mass of $4012 \mathrm{MeV}$. Futher heavy quark spin symmetry partners, up to six, can be derived from the additional assumption that the $X(3915)$ is also a $\mathrm{D}^{*} \overline{\mathrm{D}}^{*}$ bound state.
\end{abstract}

Sixth International Conference on Quarks and Nuclear Physics,

April 16-20, 2012

Ecole Polytechnique, Palaiseau, Paris

\footnotetext{
* Speaker.
} 


\section{Introduction}

Hadronic molecules - bound states between two hadrons - are a natural and well-grounded theoretical expectation of hadronic physics. The original argumentation by Voloshin and Okun [1] states that, in analogy with the traditional meson exchange picture of nuclear forces, two heavy hadrons can also exchange light mesons (provided they contain a light quark). The resulting hadronic force may eventually be strong enough as to bind the two hadrons together. Of course, the exact location of these states is difficult to predict a priori: we do not know which are the couplings of the exchanged mesons with the heavy hadrons, not to mention the problem of the form factors in the meson-hadron vertices. However there are regularities and we can always rely on guesswork to determine the approximate location of heavy hadron bound states. As noticed by Ericson and Karl [2], hadronic molecules will be more bound (i.e. more probable) the heavier the hadrons. In a different but converging line, Törnqvist [3] studied the possibility of bound states of pseudoscalar and vector heavy mesons in a simple potential model consisting of the one pion exchange (OPE) potential regularized with a monopolar form factor. The conclusion in both cases is that the lightest system to be bound is the charmed meson-antimeson one. With hindsight we see that the results from these early speculations pointed in the right direction.

The discovery of the $X(3872)$ resonance [4] might prove to be the first example of a molecular state. With a mass of $3871.68 \pm 0.17 \mathrm{MeV}$, this state lies extremely close to the $\mathrm{D}^{0} \mathrm{D}^{0 *}$ threshold $(3871.79 \pm 0.21 \mathrm{MeV})[5]$. The molecular interpretation is thus very appealing. However, as of yet there is at most circumstantial evidence on the molecular nature of the $X(3872)$, and other possibilities (tetraquark, $c \bar{c}$, hadrocharmonium) are not excluded. In fact, we do not even know for sure the $J^{p c}$ quantum numbers of the $X(3872)$. They are either $1^{++}$or $2^{-+}[6]$, of which only $1^{++}$ is compatible with a meson-antimeson bound state. Probably, the strongest hint for the molecular nature of the $X(3872)$ is the isospin violating branching ratio [7]

$$
\frac{\Gamma\left(X(3872) \rightarrow J / \Psi \pi^{+} \pi^{-} \pi^{0}\right)}{\Gamma\left(X(3872) \rightarrow J / \Psi \pi^{+} \pi^{-}\right)}=0.8 \pm 0.3,
$$

which is easily explained in the molecular picture, as shown by the first time by Gamermann et al. $[8,9]$, but apparently incompatible with competing explanations (see e.g. the interesting analysis of Ref. [10]).

In this contribution, we will explore the role of heavy quark spin symmetry (HQSS) in heavy meson-antimeson bound states within the effective field theory (EFT) framework. As we will see, HQSS constrains the interactions of heavy mesons in a very specific way and predicts the existence of HQSS partners of the $X(3872)$ (if it turns out to be molecular). We will also find that pion exchanges play a relatively minor role in the low energy description of the $X(3872)$ or the recently discovered $Z_{b}(10610)$ and $Z_{b}(10650)$ [11]. This is very interesting in the sense that it expands the range of applicability of the simplest EFT descriptions (i.e. purely contact theories) from just a few $\mathrm{MeV}$ around the open charm (bottom) thresholds up to several tens of $\mathrm{MeV}$.

\section{The Effective Field Theory Description of Hadronic Molecules}

Effective field theories are generic and systematic frameworks for the description of low energy processes. They are the most adequate theoretical tool for dealing with systems for which a 
higher energy description is impractical. Building EFTs is straightforward: we first identify the fields and symmetries that are relevant at low energies and then construct all the interactions that involve the previous fields and are consistent with the previous symmetries. Of course, this idea is only useful provided there is a separation of scales between the low energy physics we are interested in (the scale $Q$ ) and the high energy physics we do not know how to cope with (the scale $\Lambda_{0}$ ). This condition is very important: if there is no scale separation, we end up with an infinite number of interactions depending on an infinite number of coupling constants and the EFT has no predictive power whatsoever. However, if $\Lambda_{0} \gg Q$ we can order the effective interactions according to their relative importance at low energies. The principle by which this ordering is done is called power counting. If we are interested in the arbitrary quantity $A$, we expand it as

$$
A\left(Q, \Lambda_{0}\right)=\sum_{v}\left(\frac{Q}{\Lambda_{0}}\right)^{v} \hat{A}^{(v)}(Q)
$$

where each new term in the expansion is suppressed by an additional $Q / \Lambda_{0}$ factor. The EFT is now manageable: we consider only the finite number of contributions with $v<v_{\max }$, and the results will be accurate up to a relative uncertainty of $\left(Q / \Lambda_{0}\right)^{V_{\max }+1}$.

In the case of heavy meson-antimeson molecules the low energy fields are the $\mathrm{P}$ and $\mathrm{P}^{(*)}$ pseudoscalar and vector heavy mesons (plus the respective antimeson) fields and the pions that these mesons can exchange. The relevant symmetries are heavy quark spin symmetry (HQSS) [12, 13] for the heavy mesons and broken chiral symmetry for the pions and their interactions. The identification of the lowest order diagrams is not unique and depends on the properties of the system we want to describe ${ }^{1}$. We choose here the EFT expansion that is generated if there are low lying meson-antimeson bound states such as the $X$ (3872), for which the leading order (LO) interaction only contain two independent contact range interactions (i.e. four meson vertices). The power counting assignment for these two vertices is $v=-1$ (or $Q^{-1}$ ) and all other diagrams are subleading. The one pion exchange (OPE) potential, for example, enters at order $Q^{0}$, while particle coupled channel effects of the type $\mathrm{P} \overline{\mathrm{P}} \rightarrow \mathrm{P}^{*} \overline{\mathrm{P}}^{*}$ enter at order $Q^{1}$. The resulting EFT is the heavy meson counterpart of the Kaplan, Savage and Wise power counting for two nucleon systems [15, 16] (it can also be considered an upgrade of X-EFT [17] to a larger energy window). For a more detailed account of the previous ideas we refer the reader to Ref. [18].

Even though in the previous paragraph we have commented it very casually, the subleading (i.e. perturbative) character of the OPE potential in heavy meson molecules is a non-trivial and interesting result. According to naive expectations, OPE is expected to become non-perturbative at a binding energy of the order of $m_{\pi}^{2} / M_{P}$, about $10 \mathrm{MeV}(4 \mathrm{MeV})$ in the charm (bottom) sector. However, by adapting a series of techniques of nuclear EFT [19] to heavy meson-antimeson molecules we find that the conclusion that OPE is perturbative is valid for a range of binding energies much larger than expected, up to $100 \mathrm{MeV}$ in the isoscalar charm sector, and $50 \mathrm{MeV}$ in the isovector bottom sector [18]. This entails a remarkable simplifications of the EFT calculations at LO, for which a purely contact theory can be used up to relatively large binding energies.

\footnotetext{
${ }^{1}$ A more formal account involves the analysis of the renormalization group evolution of the EFT operators and states that there are different EFT expansions corresponding to different fixed points of the renormalization group [14].
} 


\section{The HQSS Partners of the $X(3872)$}

The final form of the $\mathrm{LO}\left(Q^{-1}\right)$ effective potential in momentum space reads

$$
\begin{aligned}
V_{\mathrm{P} \overline{\mathrm{P}}}^{(-1)}\left(\vec{q}, 0^{++}\right) & =C_{0 a}, \\
V_{\mathrm{P}^{*} \mathrm{P} / \mathrm{P}^{*}}^{(-1)}\left(\vec{q}, 1^{+-}\right) & =C_{0 a}-C_{0 b}, \\
V_{\mathrm{P}^{*} \mathrm{P} / \mathrm{P}^{*}}^{(-1)}\left(\vec{q}, 1^{++}\right) & =C_{0 a}+C_{0 b}, \\
V_{\mathrm{P}^{*} \overline{\mathrm{P}}^{*}}^{(-1)}\left(\vec{q}, 0^{++}\right) & =C_{0 a}-2 C_{0 b}, \\
V_{\mathrm{P}^{*} \overline{\mathrm{P}}^{*}}^{(-1)}\left(\vec{q}, 1^{+-}\right) & =C_{0 a}-C_{0 b}, \\
V_{\mathrm{P}^{*} \overline{\mathrm{P}}^{*}}^{(-1)}\left(\vec{q}, 2^{++}\right) & =C_{0 a}+C_{0 b},
\end{aligned}
$$

where the subscripts indicate the particle channel $\left(\mathrm{P} \overline{\mathrm{P}}, \mathrm{P}^{*} / \mathrm{P}^{*} \overline{\mathrm{P}}, \mathrm{P}^{*} \overline{\mathrm{P}}^{*}\right)$. The EFT potential must be regulated with an arbitrary regulator function, which we choose to be a gaussian regulator. For the cut-off we use the values $\Lambda=500 / 1000 \mathrm{MeV}$, which are of the order of the natural high energy scale for hadronic molecules. Owing to the renormalization group invariance properties of the EFT formulation, we expect the cut-off uncertainty to be a higher order effect (i.e. at least $Q^{0}$ ). The regularized potential is then iterated in the Lippmann-Schwinger equation $T=V+V G_{0} T$, where we will search for poles of the T-matrix as they correspond to bound states (see Refs. [20, 21] for details).

As can be seen, the degree of symmetry of the effective potential is remarkable. In particular, we stress that

$$
\begin{aligned}
V_{\mathrm{P}^{*} \overline{\mathrm{P}} / \mathrm{P}^{*}}^{(-1)}\left(\vec{q}, 1^{+-}\right) & =V_{P^{*} \bar{P}^{*}}^{(-1)}\left(\vec{q}, 1^{+-}\right), \\
V_{\mathrm{P}^{*} \overline{\mathrm{P}} / \mathrm{P}^{*}}^{(-1)}\left(\vec{q}, 1^{++}\right) & =V_{P^{*} \bar{P}^{*}}^{(-1)}\left(\vec{q}, 2^{++}\right) .
\end{aligned}
$$

The first line in the equation above explains why the $Z_{b}(10610)$ and $Z_{b}(10650)$ resonances show the same energy shift with respect to the $\mathrm{B}^{*}$ and $\mathrm{B}^{*} \overline{\mathrm{B}}^{*}$ thresholds (in this respect, see the discussions of Refs. [22, 23]). However, if we are considering the charm sector, we may be more interested in the second line, which states that the EFT potential for the $1^{++} \mathrm{P}^{*} \overline{\mathrm{P}} / \mathrm{P}^{*}$ and $2^{++} \mathrm{P}^{*} \overline{\mathrm{P}}^{*}$ channels is identical. From this we can derive the following consequence: if the $X(3872)$ is a $\mathrm{DD}^{*} / \mathrm{D}^{*} \overline{\mathrm{D}}$ bound state with quantum numbers $1^{++}$, then we should expect a $2^{++} \mathrm{D}^{*} \overline{\mathrm{D}}^{*}$ molecule with a mass of $4012 \mathrm{MeV}$. We call this state the $X(4012)$.

The prediction of the $X(4012)$ is model independent and relies only on HQSS and the assumption that the $X(3872)$ is indeed molecular. However, there are subleading order corrections that can spoil this prediction, namely (i) the effect of the OPE potential and (ii) HQSS violations. The energy shift induced by the OPE potential is small, moving the $2^{++} \mathrm{D}^{*} \overline{\mathrm{D}}^{*}$ state up to $4014-4015 \mathrm{MeV}$. The larger effect corresponds to HQSS violations, which amount to a $\pm\left(\Lambda_{\mathrm{QCD}} / m_{c}\right)$ relative correction to the potential in the $2^{++}$channel with respect to its HQSS expectation. This implies that the mass of the state can change from 4003 to $4016 \mathrm{MeV}$.

Apart from the $X(3872)$ and $X(4012)$, HQSS is compatible with the existence of up to six charmed meson-antimeson bound states. Predicting them requires the determination of the two LO counterterms, $C_{0 a}$ and $C_{0 b}$, which in turn requires the identification of a molecular state with 


\begin{tabular}{|c|c|c|c|c|}
\hline \hline$J^{P C}$ & $\mathrm{H} \overline{\mathrm{H}}$ & $E(\Lambda=0.5 \mathrm{GeV})$ & $E(\Lambda=1 \mathrm{GeV})$ & $\operatorname{Exp}(\mathrm{PDG}[5])$ \\
\hline $0^{++}$ & $D \bar{D}$ & $3706 \pm 10$ & $3712_{-17}^{+13}$ & - \\
\hline $1^{++}$ & $D^{*} \bar{D}$ & Input & Input & 3872 \\
$1^{+-}$ & $D^{*} \bar{D}$ & $3814 \pm 17$ & $3819_{-27}^{+24}$ & - \\
\hline $0^{++}$ & $D^{*} \bar{D}^{*}$ & Input & Input & 3917 \\
$1^{+-}$ & $D^{*} \bar{D}^{*}$ & $3953 \pm 17$ & $3956_{-28}^{+25}$ & 3942 \\
$2^{++}$ & $D^{*} \bar{D}^{*}$ & $4012 \pm 3$ & $4012_{-9}^{+4}$ & - \\
\hline
\end{tabular}

Table 1: The HQSS molecular partners of the $X(3872)$ and $X(3915)$. The bound states are computed from the LO effective potential of Eqs. (3.1 - 3.6), where the counterterms are fixed by reproducing the location of the $X(3872)$ and $X(3915)$ resonances. The calculations are performed for two values of the cut-off, and the error in the masses of the states are computed by assuming a $\Lambda_{\mathrm{QCD}} / m_{c}$ relative violation of HQSS in the countertems. Further details can be consulted in Ref. [21].

quantum numbers other than $1^{++}$or $2^{++}$. There are two promising candidates, the $X(3915)$ [24] and $X(3940)$ [25], from which the first has been already theorized to be molecular [26]. However, we momentarily discard the $X(3940)$ : the production mechanism for this state is $e^{+} e^{-} \rightarrow$ $J / \Psi X(3940)$, suggesting a positive C-parity state (assuming one intermediate virtual photon). At the same time this resonance decays mostly to $\mathrm{DD}^{*}$, which is only compatible with a $1^{+-}$bound state, thus contradicting the previous C-parity assignment. Therefore, we theorize the $X(3915)$ to be a $0^{++} \mathrm{D}^{*} \overline{\mathrm{D}}^{*}$ molecule, a choice that leads to the prediction of a total of six HQSS molecular partners that we list in Table 1 . We have checked the results against several uncertainty sources, in particular (i) OPE, (ii) HQSS violations and (iii) particle coupled channel effects. Contrary to common wisdom, but as expected from the analysis of Ref. [18], OPE effects are unimportant, changing the position of the states by a few MeV only. This is true even for the $X(3915)$, which has a binding energy of $100 \mathrm{MeV}$. The effect of HQSS violations are already included in Table 1. Finally, the explicit inclusion of particle coupled channels changes the position of the $0^{++}\left(1^{+-}\right)$ states by around $40 \mathrm{MeV}(30 \mathrm{MeV})$, but does not alter substantially the pattern of these states, which seems to be quite robust. It is also interesting to mention that most of the states of Table 1 have also been predicted in other unrelated theoretical frameworks [27, 28, 29, 30].

To summarize, we have analyzed the role of the OPE potential and HQSS in heavy mesonantimeson molecules from the EFT perspective. Surprisingly, the effect of the OPE potential is far weaker than expected, even for tightly bound states. That is, the lowest order EFT description of molecular states consists solely of contact interactions, with pions entering as a subleading perturbation [18]. The consequences of HQSS are very interesting too, as this symmetry predicts the existence of a new, so far undiscovered $2^{++}$partner of the $X(3872)$, which we call the $X(4012)$. If we also assume the $X(3915)$ to be a $0^{++}$molecule, then we end up predicting three additional states at 3710,3820 and $3950 \mathrm{MeV}$ respectively [21].

Acknowledgments: this work was supported by the DGI under contract FIS2011-28853-C0202, the Generalitat Valenciana contract PROMETEO/2009/0090, the Spanish Ingenio-Consolider 2010 Program CPAN (CSD2007-00042) and the EU Research Infrastructure Integrating Initiative HadronPhysics2. 


\section{References}

[1] M. B. Voloshin and L. B. Okun, JETP Lett. 23 (1976) 333 [Pisma Zh. Eksp. Teor. Fiz. 23 (1976) 369].

[2] T. E. O. Ericson and G. Karl, Phys. Lett. B 309 (1993) 426.

[3] N. A. Tornqvist, Z. Phys. C 61 (1994) 525.

[4] S. K. Choi et al. [Belle Collaboration], Phys. Rev. Lett. 91 (2003) 262001.

[5] K. Nakamura et al. [Particle Data Group Collaboration], J. Phys. G G 37 (2010) 075021.

[6] A. Abulencia et al. [CDF Collaboration], Phys. Rev. Lett. 98 (2007) 132002.

[7] S. -K. Choi, S. L. Olsen, K. Trabelsi, I. Adachi, H. Aihara, K. Arinstein, D. M. Asner and T. Aushev et al., Phys. Rev. D 84 (2011) 052004.

[8] D. Gamermann and E. Oset, Phys. Rev. D 80 (2009) 014003.

[9] D. Gamermann, J. Nieves, E. Oset and E. Ruiz Arriola, Phys. Rev. D 81 (2010) 014029.

[10] C. Hanhart, Y. .S. Kalashnikova, A. E. Kudryavtsev and A. V. Nefediev, Phys. Rev. D 85 (2012) 011501.

[11] A. Bondar et al. [Belle Collaboration], Phys. Rev. Lett. 108, 122001 (2012).

[12] N. Isgur and M. B. Wise, Phys. Lett. B 232 (1989) 113.

[13] N. Isgur and M. B. Wise, Phys. Lett. B 237 (1990) 527.

[14] M. C. Birse, J. A. McGovern and K. G. Richardson, Phys. Lett. B 464 (1999) 169.

[15] D. B. Kaplan, M. J. Savage and M. B. Wise, Phys. Lett. B 424 (1998) 390.

[16] D. B. Kaplan, M. J. Savage and M. B. Wise, Nucl. Phys. B 534 (1998) 329.

[17] S. Fleming, M. Kusunoki, T. Mehen and U. van Kolck, Phys. Rev. D 76, 034006 (2007).

[18] M. Pavon Valderrama, Phys. Rev. D 85 (2012) 114037.

[19] M. C. Birse, Phys. Rev. C 74 (2006) 014003.

[20] J. Nieves and M. Pavon Valderrama, Phys. Rev. D 84 (2011) 056015.

[21] J. Nieves and M. Pavon Valderrama, arXiv:1204.2790 [hep-ph].

[22] M. B. Voloshin, Phys. Rev. D 84 (2011) 031502.

[23] T. Mehen and J. W. Powell, Phys. Rev. D 84 (2011) 114013.

[24] S. Uehara et al. [Belle Collaboration], Phys. Rev. Lett. 104 (2010) 092001.

[25] K. Abe et al. [Belle Collaboration], Phys. Rev. Lett. 98 (2007) 082001.

[26] T. Branz, T. Gutsche and V. E. Lyubovitskij, Phys. Rev. D 80 (2009) 054019.

[27] L. Maiani, F. Piccinini, A. D. Polosa and V. Riquer, Phys. Rev. D 71 (2005) 014028.

[28] D. Gamermann, E. Oset, D. Strottman and M. J. Vicente Vacas, Phys. Rev. D 76 (2007) 074016.

[29] D. Gamermann and E. Oset, Eur. Phys. J. A 33 (2007) 119.

[30] R. Molina and E. Oset, Phys. Rev. D 80 (2009) 114013. 